\title{
MÉTODOS PARA AVALIAÇÃO DO VIGOR DE SEMENTES DE SOJA, INCLUINDO A ANÁLISE COMPUTADORIZADA DE IMAGENS ${ }^{1}$
}

\author{
JULIO MARCOS FILHO², ANA LÚCIA PEREIRA KIKUTI³, LIANA BAPTISTA DE LIMA³.
}

\begin{abstract}
RESUMO - A velocidade e uniformidade de emergência de plântulas, determinantes do sucesso do estabelecimento do estande, representam etapas essenciais para a obtenção de alta produtividade na cultura da soja, de modo que o desenvolvimento de procedimentos para avaliar o potencial fisiológico tem recebido atenção contínua da pesquisa em Tecnologia de Sementes. O objetivo deste trabalho foi o de verificar a eficiência de um sistema computadorizado para análise de imagens de plântulas, em comparação com testes recomendados para determinação do vigor de sementes de soja como testes de tetrazólio, envelhecimento acelerado, condutividade elétrica e crescimento de plântulas,. Para tanto, lotes de sementes dos cultivares Pintado e TMG 113RR foram avaliados quanto à germinação (25oC aos 4 e 7 dias), envelhecimento acelerado tradicional (41oC durante $48 \mathrm{~h}$ ), envelhecimento acelerado com solução saturada de sal (41oC durante 72 h) e emergência de plântulas. O sistema de análise computadorizada de imagens de plântulas (SVIS $®$ ), desenvolvido pela Ohio State University/EUA, forneceu dados referentes ao índice de vigor, à uniformidade de desenvolvimento e ao crescimento de plântulas, tanto aos três dias após a semeadura do teste de germinação sob condições ótimas como após o envelhecimento acelerado com solução de sal. O período experimental compreendeu três épocas de testes com intervalo aproximado de 30 dias. Constatou-se que o SVIS ${ }^{\circ}$ é suficientemente sensível para avaliação do vigor de sementes de soja, com eficiência comparável à do teste de envelhecimento acelerado, constituindo alternativa interessante para a obtenção de informações consistentes sobre o potencial fisiológico.
\end{abstract}

Termos para indexação: Glycine max, análise de sementes, germinação, potencial fisiológico

\section{PROCEDURES FOR EVALUATION OF SOYBEAN SEED VIGOR, INCLUDING AN AUTOMATED COMPUTER IMAGING SYSTEM}

\begin{abstract}
ABSTRAC - Speed and uniformity of seedling emergence and stand establishment are crucial issues to support high productivity soybean crops. As a consequence, the development and improvement of reproducible procedures to evaluate the physiological attributes of seed quality is a continuing need of the seed industry. Soybean seed vigor has been primarily evaluated by the accelerated aging, electrical conductivity, tetrazolium and seedling growth tests, but there are still opportunities to improve methodology. The objective of this study was to verify the accuracy of an automated system for seedling evaluation (SVIS $\AA$ ) to assess soybean seed vigor in comparison to the information from accepted vigor tests and seedling emergence results. Five seed lots each of two cultivars (Pintado
\end{abstract}

${ }^{1}$ Submetido em 09/09/2008. Aceito para publicação em 19/01/2009.

${ }^{2}$ Eng. Agr., Dr., Professor Titular, Departamento de Produção Vegetal, USP/ ESALQ, Caixa Postal 9, 13418-900 Piracicaba, SP. Bolsista do CNPq. jmarcos@esalq.usp.br

${ }^{3}$ Eng. Agr., Dr., Programa de Pós-doutoramento, Departamento de Produção Vegetal, USP/ESALQ 
and TMG 113RR) were subjected to standard germination, traditional accelerated aging (41oC and $100 \%$ R.H. for $48 \mathrm{~h}$ followed by germination at $25 \mathrm{oC}$ for 4 days), saturated salt accelerated aging SSAA (41oC and 75\% R.H. for 72h), and field seedling emergence. The Seed Vigor Imaging System $(\mathrm{SVIS} \circledast$ ) parameters comprised mean seedling growth, uniformity and vigor index in images of 3-day old seedlings after germination or SSAA tests. The experimental period comprised three evaluation times at 30-day intervals. Results showed that the information provided by the SVIS $®$ analyses represented a rapid and consistent alternative for soybean seed vigor evaluation with efficiency compared to the accelerated aging test.

Index terms: Glycine max, seed analysis, germination, physiological potential

\section{INTRODUÇÃO}

Os testes de vigor têm sido utilizados principalmente para identificar diferenças associadas ao desempenho de lotes de sementes durante o armazenamento ou após a semeadura, procurando destacar lotes com maior eficiência para o estabelecimento do estande sob ampla variação das condições de ambiente.

A avaliação do vigor de sementes constitui providência fundamental para o sucesso da produção de sementes de soja, reconhecidas por sua sensibilidade à deterioração e a práticas de manejo menos adequadas após a maturidade (Marcos Filho, 1999a). Vários testes têm sido recomendados para a avaliação do vigor de sementes de soja, destacando-se os de envelhecimento acelerado, tetrazólio, condutividade elétrica, crescimento de plântulas, classificação do vigor de plântulas (Vieira et al., 2003). As instituições públicas de pesquisa e as universidades têm se preocupado principalmente com a condução de pesquisas visando à adequação de procedimentos para a determinação do vigor, a identificação de sua eficiência e a tentativa de padronização para que as empresas produtoras possam utilizar essas informações para a composição de programas internos de controle de qualidade (Vieira, 1999).

Por outro lado, embora o procedimento básico para a condução de vários testes de vigor esteja estabelecido e produza resultados confiáveis, sempre há espaço para aprimoramento da metodologia ou inclusão de alternativas inovadoras. O primeiro caso pode ser exemplificado pela possibilidade de redução do período de pré-condicionamento para a condução do teste de tetrazólio em sementes de soja, sem prejudicar sua eficiência (Costa et al., 2008), enquanto o uso da análise automatizada de imagens de sementes e plântulas ilustra uma iniciativa associada à modernidade.

A literatura tem documentado vários estudos envolvendo a análise de imagens em tecnologia de sementes, principalmente os raios-X e a análise de imagens de plântulas, visando estabelecer sua relação com o vigor de sementes. Neste sentido, McCormac et al. (1990) demonstrou a viabilidade do uso de um sistema automático para a determinação do vigor de sementes de couve-flor, mediante a avaliação individual do comprimento da raiz primária de plântulas. Mais recentemente, Sako et al. (2001) desenvolveram um programa destinado à determinação do vigor de sementes de alface, mediante a análise computadorizada de imagens de plântulas (Seed Vigor Imaging System - SVIS ${ }^{\circledR}$ ). O sistema foi adaptado com sucesso para outras espécies, como soja (Hoffmaster et al., 2003), milho (Otoni e McDonald, 2005 ), melão (Marcos Filho et al., 2006), permitindo o cálculo de índice de vigor, grau de uniformidade de desenvolvimento e avaliação do comprimento de plântulas ou de suas partes. McDonald et al. (2003) discutiram as diferentes alternativas para aplicação do sistema automatizado para a determinação do vigor de sementes e as amplas possibilidades de sucesso na obtenção de informações consistentes. Essa metodologia tem sido utilizada rotineiramente por laboratórios de entidades certificadoras de sementes e de empresas produtoras nos Estados Unidos da América, particularmente no estado de Ohio, sendo considerado procedimento promissor para avaliação segura do vigor de sementes de várias espécies cultivadas.

O sistema permite efetuar a análise relativamente rápida de plântulas (geralmente menos de 60 segundos para avaliação de 50 plântulas em um rolo de papel-toalha), representando economia significativa de tempo, em comparação com a maioria dos testes para avaliação rotineira da qualidade das sementes. Ao mesmo tempo, o potencial de uso do sistema não se restringe à comparação direta do vigor de diferentes lotes mediante a análise do crescimento de plântulas; é provável a possibilidade de sua introdução como complemento de outros 
testes como, por exemplo, de envelhecimento acelerado (Sekharan, 2005; Otoni et al., 2008). Neste teste, a avaliação da reação de amostras de sementes aos efeitos da temperatura e umidade relativa elevada poderia ser efetuada com a análise computadorizada de plantas, o mesmo acontecendo com as sementes submetidas ao condicionamento fisiológico, com real possibilidade de padronização da metodologia.

A determinação do potencial fisiológico de sementes mediante a comparação do crescimento de plântulas ou de suas partes é um procedimento de eficiência comprovada (Woodstock, 1969; Steiner, 1990; Nakagawa, 1999) para detectar diferenças entre lotes de sementes e estabelecer relações com a emergência de plântulas em campo. Portanto, a avaliação automatizada do crescimento de plântulas, reduzindo a subjetividade das análises baseadas na observação visual do analista, pode constituir - se em um avanço significativo rumo à padronização da metodologia

Diante do exposto, o presente trabalho teve como objetivo verificar a eficiência do sistema computadorizado de avaliação do vigor de sementes (SVIS ${ }^{\circledR}$ ), em comparação com procedimentos comumente utilizados em sementes de soja, bem como verificar a possibilidade de utilização desse sistema para a interpretação de resultados do teste de envelhecimento acelerado.

\section{MATERIAL E MÉTODOS}

A pesquisa foi conduzida nos laboratórios de Análise de Sementes e de Análise de Imagens, Departamento de Produção Vegetal/Escola Superior de Agricultura Luiz de Queiroz/Universidade de São Paulo, em maio/agosto de 2008. Utilizaram-se os cultivares TMG 113RR e Pintado, cada qual representado por cinco lotes de sementes com germinação superior a $85 \%$, produzidos em $2007 / 2008$ por Sementes Adriana, em Rondonópolis/MT.

As amostras recebidas foram mantidas em condições normais de ambiente em laboratório e submetidas à determinação do grau de umidade (método da estufa, a $105^{\circ} \mathrm{C}$ ) e a testes de germinação e de vigor (envelhecimento acelerado tradicional e com solução de $\mathrm{NaCl}$; SVIS; SVIS após o envelhecimento acelerado e emergência de plântulas), com intervalos aproximados de 30 dias. As sementes de 'TMG 113R' foram avaliadas em duas épocas e, as de 'Pintado', em três épocas experimentais. Adotaram-se os seguintes procedimentos:

Germinação: conduzido com quatro repetições de 50 sementes para cada lote e cultivar, em rolos de papel-toalha, a $25^{\circ} \mathrm{C}$; o umedecimento foi efetuado com quantidade de água equivalente e 2,5 vezes o peso do substrato seco. Os testes foram interpretados aos 4 e aos 7 dias após a semeadura, de acordo com os critérios estabelecidos nas Regras para Análise de Sementes (BRASIL, 1992). Os resultados foram expressos em percentagem de plântulas normais para cada lote.

Envelhecimento acelerado (tradicional e com solução saturada de sal): foram utilizadas caixas de plástico (11 cm x $11 \mathrm{~cm}$ x $3 \mathrm{~cm}$ ) como compartimentos individuais. A umidade relativa no interior dessas caixas foi obtida pela adição de $40 \mathrm{ml}$ de água (para a obtenção de, aproximadamente, 100\% U.R. do ar) ou de solução saturada de cloreto de sódio (ambiente com $75 \%$ U.R.) ao fundo de cada caixa, conforme descrição efetuada por Marcos Filho et al. (2000). As amostras de cada lote foram distribuidas de modo a constituir camada única tomando toda a superfície da tela metálica suspensa no interior de cada caixa. O envelhecimento acelerado tradicional (EAT) foi conduzido a $41^{\circ} \mathrm{C}$ durante 48 horas; no procedimento com solução saturada de sal (EASS), as sementes permaneceram durante 72 horas no interior da câmara B.O.D. seguindo-se, em ambas as situações, a condução de teste de germinação a $25^{\circ} \mathrm{C}$, durante quatro dias; foi computada a percentagem média de plântulas normais para cada lote e cultivar.

Emergência de plântulas: foram utilizadas quatro repetições de 50 sementes por lote e cultivar, distribuídas na superfície de uma camada de $5 \mathrm{~cm}$ de areia colocada em caixas de plástico $(32 \mathrm{~cm} \times 28 \mathrm{~cm} \times 10 \mathrm{~cm})$. Após a semeadura, a cobertura foi efetuada com uma camada de $2 . \mathrm{cm}$ de areia. $\mathrm{O}$ umedecimento do substrato foi efetuado com quantidade de água correspondente a $60 \%$ da capacidade de retenção. As caixas foram expostas a temperatura normal de ambiente, em laboratório, durante 10 dias, quando foi determinada a percentagem média de emergência de plântulas para cada lote e cultivar.

\section{SVIS ${ }^{\circledR}$ para avaliação do vigor de sementes:}

a) Captura e processamento das imagens:

As plântulas foram obtidas de quatro repetições de 25 sementes para cada lote, colocadas para germinar em duas fileiras situadas no terço superior da superfície do papeltoalha, a $25^{\circ} \mathrm{C}$, durante três dias. Em seguida, as imagens foram captadas em scanner HP Scanjet 2004, montado de maneira invertida no interior de uma caixa de alumínio com $60 \mathrm{~cm}$ x $50 \mathrm{~cm} \times 12 \mathrm{~cm}$ e operado por software Photosmart, com resolução de 98 dpi.

As plântulas normais identificadas em cada repetição (no máximo, 25) foram transferidas do papel-toalha para uma folha de cartolina de coloração azul escuro com $30 \mathrm{~cm}$ 
x $22 \mathrm{~cm}$ (correspondente ao tamanho da área útil atingida pelo scanner), colocada sobre a plataforma interna da caixa metálica (Figura 1). As imagens escaneadas foram salvas em pasta específica do disco rígido e, em seguida, analisadas pelo software Seed Vigor Imaging System $\left(\right.$ SVIS $\left.^{\circledR}\right)$ instalado em computador Pentium IV, com CPU de 2,0 GHz, $768 \mathrm{MB}$ RAM e HD de $40 \mathrm{~Gb}$, operado por sistema Windows XP Profissional.

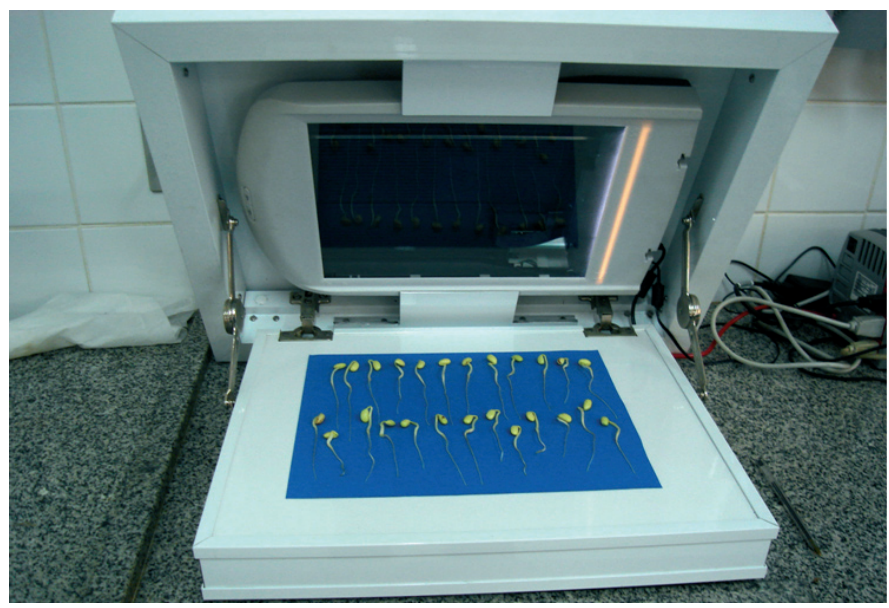

FIGURA 1. Visão geral do scanner montado de maneira invertida, no interior de caixa de alumínio, no momento da aquisição da imagem de uma amostra de plântulas de soja.

Nessa análise, o eixo raiz/hipocótilo de cada plântula é marcado em vermelho e, para a maioria das plântulas o eixo foi identificado corretamente; houve, no entanto, necessidade de efetuar correções manuais de erros (com auxílio do "mouse"), como a complementação de uma parte de plântulas marcadas parcialmente e tanto a exclusão de plântulas com anormalidades extremas como a inclusão de plântulas não computadas. Após a análise e avaliação de cada plântula (Figura 2), o software gera automaticamente valores numéricos referentes a um índice de vigor (valores de 0 a 1000 , diretamente proporcionais ao vigor) e à uniformidade de desenvolvimento (também de 0 a 1000).

Os valores do índice de vigor são baseados na rapidez e uniformidade de desenvolvimento das plântulas da amostra, em relação ao máximo valor possível para plântulas de soja com 3 dias de idade, parâmetros estabelecidos na programação do software (Hoffmaster et al., 2003). Por outro lado, a uniformidade é estabelecida em função do desvio em relação ao desenvolvimento padrão de plântulas com 3 dias de idade, também programado no software.

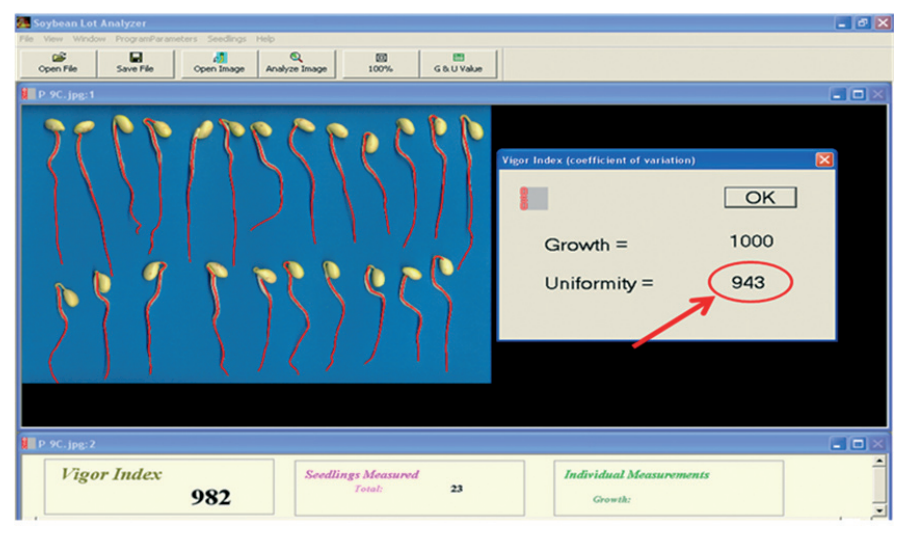

FIGURA 2. Imagens analisadas de uma amostra de plântulas de soja, com indicações da marcação do eixo raiz/hipocótilo (em vermelho), do índice de vigor (982) e da uniformidade (943).

Depois de computados esses valores, o cursor é posicionado sobre cada plântula e acionado, indicando automaticamente o valor correspondente ao comprimento da plântula demarcada, em milímetros; esses valores são apresentados em janela situada abaixo das imagens das plântulas (Figura 3). Após o processamento das imagens, foram obtidos valores médios do índice de vigor, uniformidade de desenvolvimento e comprimento de plântulas para cada lote e cultivar (Hoffmaster et al., 2005; Marcos Filho et al., 2006); como ilustração, dados obtidos para duas amostras do cv. Pintado são apresentados na Figura 4. O período compreendido entre o "escaneamento" e o final da análise de cada repetição foi, geralmente, inferior a 5 minutos.

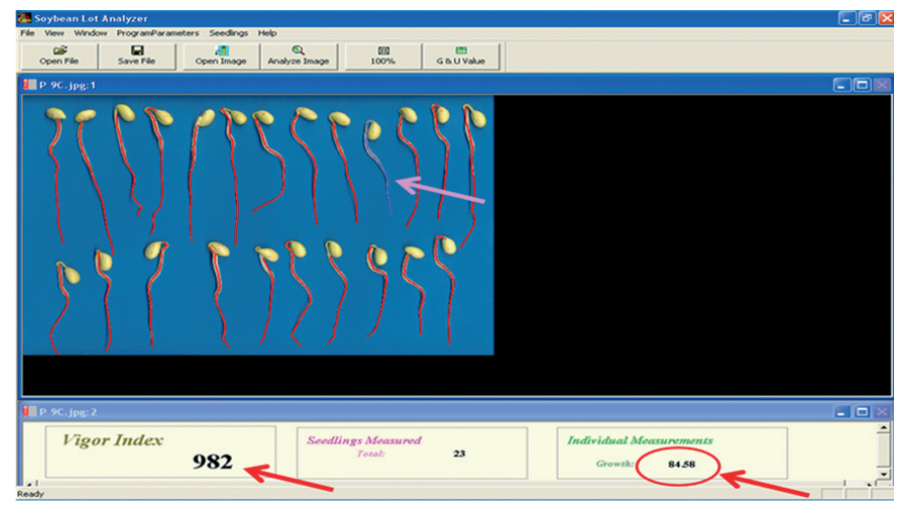

FIGURA 3. Imagens analisadas de uma amostra de plântulas de soja, com indicações da marcação do eixo raiz/hipocótilo, do índice de vigor (982) e do comprimento da plântula (mm) marcada em azul $(84,6)$. 


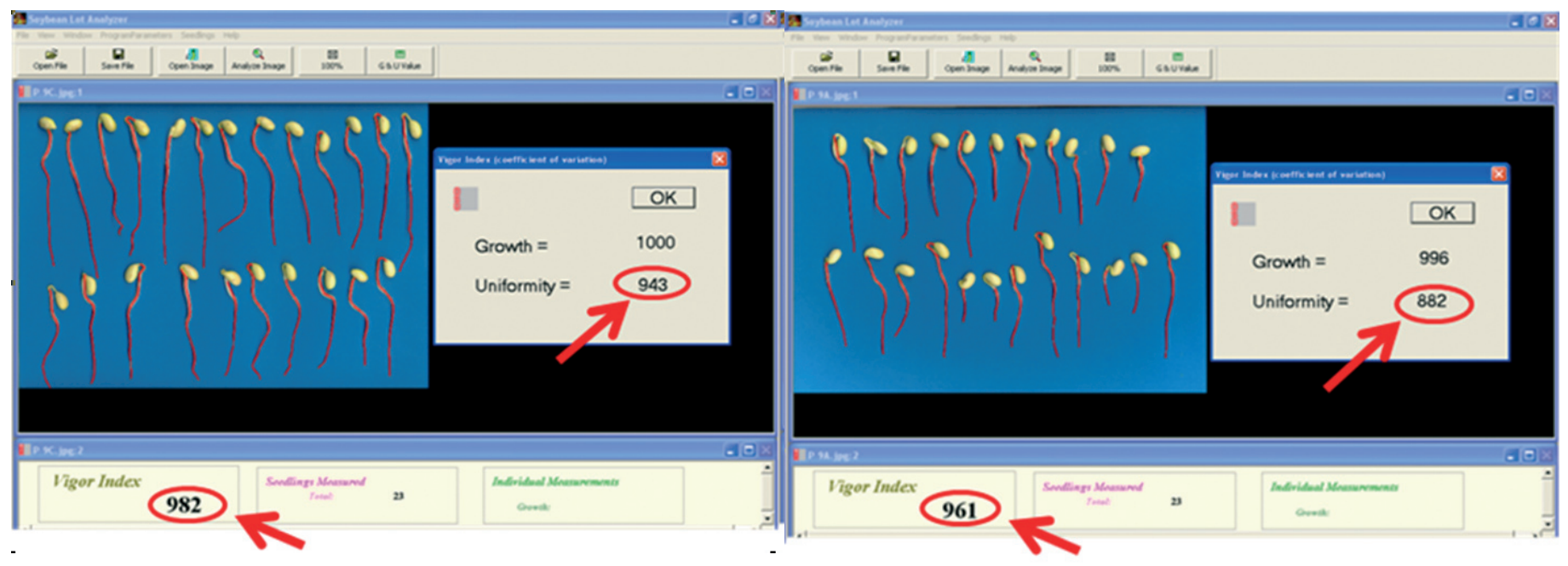

FIGURA 4. Imagens analisadas de duas amostras de plântulas de soja, cv. TMG 113R, indicando diferenças no desempenho das sementes; na amostra (A), índice de vigor $=982$ e uniformidade $=943$; na amostra (B), índice de vigor $=961$ e uniformidade $=882$

\section{SVIS $^{\circledR}$ após o envelhecimento acelerado}

As sementes foram submetidas ao envelhecimento acelerado com solução saturada de $\mathrm{NaCl}$, conforme descrição anterior. Em seguida, quatro repetições de 25 sementes de cada lote foram colocadas para germinar, em duas fileiras situadas no terço superior da superfície do papel-toalha; aos três dias após a semeadura, as plântulas foram escaneadas e analisadas com o uso do software $\mathrm{SVIS}^{\circledR}$, determinando-se índices de vigor, grau de uniformidade e comprimento de plântulas (mm) para cada lote e cultivar.

Procedimento estatístico: os dados obtidos foram submetidos à análise da variância, separadamente para cada cultivar e teste, de acordo com delineamento inteiramente casualizado. As médias foram comparadas pelo teste de Tukey $(\mathrm{p} \leq 0,05)$.

\section{RESULTADOS E DISCUSSÃO}

As análises da variância dos dados obtidos com as sementes dos dois cultivares revelaram diferenças significativas de potencial fisiológico entre lotes, em todas as épocas de testes, para a maioria dos parâmetros avaliados. Essa constatação indica que os testes conduzidos foram sensíveis a diferenças no desempenho das sementes, mesmo em lotes com poder germinativo elevado, ou seja, dentro do limite estabelecido ( $\geq 85 \%$ ) para a comercialização de sementes de soja.

O exame das Tabelas 1 e 2, correspondentes a determinações efetuadas com sementes de 'TMG 113RR', permite verificar que o lote 2 apresentou desempenho inferior nos testes de germinação, envelhecimento acelerado tradicional e com solução saturada de sal. A eficiência dos testes de envelhecimento tem sido documentada na literatura por vários autores, dentre os quais Marcos Filho et al. (2000).

A ausência de diferenças entre os lotes quanto à emergência de plântulas não foi surpreendente, pois as sementes possuíam poder germinativo elevado e as condições de ambiente foram muito favoráveis. Desta forma, considera-se que os lotes de sementes de 'TMG RR113' não apresentavam variações suficientes no vigor a ponto de se manifestar na percentagem de emergência das plântulas, sob condições adequadas.

Paralelamente, como mostra a Tabela 5, esses testes indicaram o menor potencial fisiológico das sementes do lote 10, cultivar Pintado, na terceira época de testes. Destaquese que os dados da primeira época (Tabela 3) mostraram essa mesma tendência, mas os efeitos de lotes não foram estatisticamente significativos. Na terceira época, também foi detectado o desempenho inferior das sementes do lote 10, no teste de emergência de plântulas; essa constatação pode ter sido devida ao fato das diferenças de vigor entre os lotes de 'Pintado' terem sido ligeiramente mais amplas que as verificadas para os de 'TMG 113RR'. A eficiência dos testes de vigor e as relações desses resultados com a emergência de plântulas, aqui relatados, são coerentes com as informações destacadas na literatura (Vieira, 1999; Marcos Filho, 1999b). 
TABELA 1. Valores médios obtidos em testes de germinação, primeira contagem de germinação, envelhecimento acelerado tradicional e com solução saturada de sal, emergência de plântulas eíndices de vigor, uniformidade e comprimento de plântulas de soja determinados em análises SVIS (após germinação e envelhecimento acelerado) de cinco lotes de sementes de soja, cultivar TMG 113RR, na primeira época experimental. Piracicaba, SP, 2008.

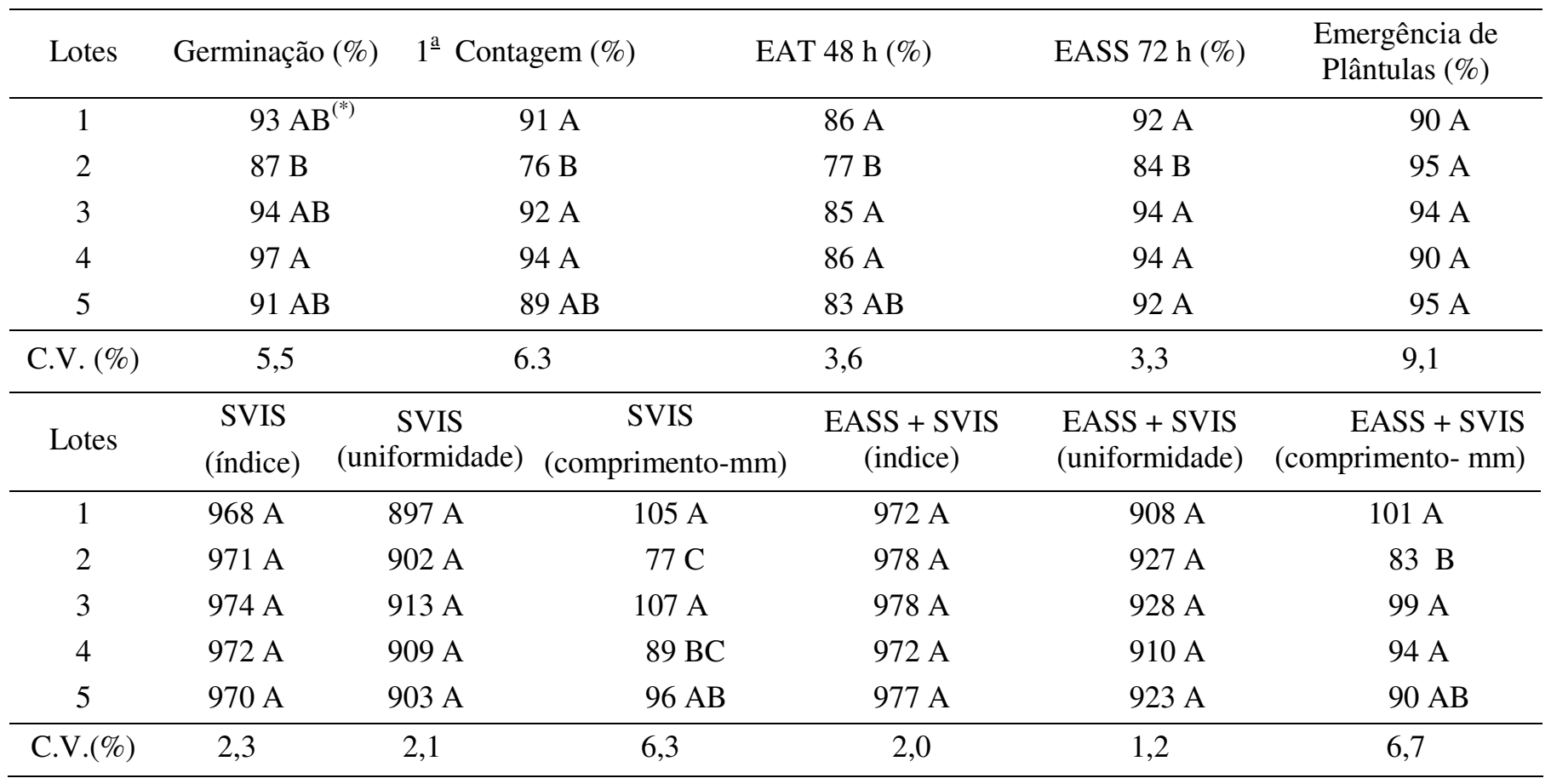

(*) Comparação de médias dentro de cada coluna (teste de Tukey, $\mathrm{p} \leq 0,05$ )

TABELA 2. Valores médios obtidos em testes de germinação, envelhecimento acelerado com solução saturada de sal, emergência de plântulas e índices de vigor, uniformidade e comprimento de plântulas de soja determinados em análises SVIS (após germinação e envelhecimento acelerado) de cinco lotes de sementes de soja, cultivar TMG 113RR, na terceira época experimental. Piracicaba, SP, 2008.

\begin{tabular}{|c|c|c|c|c|c|c|}
\hline Lotes & Germinação (\%) & \multicolumn{2}{|c|}{$1^{\mathrm{a}}$ Contagem $(\%)$} & \multicolumn{2}{|c|}{ EASS 72 h (\%) } & $\begin{array}{l}\text { Emergência de Plântulas } \\
(\%)\end{array}$ \\
\hline 1 & $90 \mathrm{AB}^{(*)}$ & \multicolumn{2}{|c|}{$87 \mathrm{~A}$} & \multicolumn{2}{|c|}{$91 \mathrm{AB}$} & $91 \mathrm{~A}$ \\
\hline 2 & $85 \mathrm{~B}$ & \multicolumn{2}{|c|}{$83 \mathrm{~A}$} & \multicolumn{2}{|c|}{$87 \mathrm{~B}$} & $96 \mathrm{~A}$ \\
\hline 3 & $91 \mathrm{AB}$ & \multicolumn{2}{|c|}{$87 \mathrm{~A}$} & \multicolumn{2}{|c|}{$93 \mathrm{~A}$} & $92 \mathrm{~A}$ \\
\hline 4 & $94 \mathrm{~A}$ & \multicolumn{2}{|c|}{$91 \mathrm{~A}$} & \multicolumn{2}{|c|}{$93 \mathrm{~A}$} & $93 \mathrm{~A}$ \\
\hline 5 & $90 \mathrm{AB}$ & \multicolumn{2}{|c|}{$86 \mathrm{~A}$} & \multicolumn{2}{|c|}{$91 \mathrm{AB}$} & $96 \mathrm{~A}$ \\
\hline C.V. $(\%)$ & 6,3 & \multicolumn{2}{|c|}{4,7} & \multicolumn{2}{|c|}{3,3} & 7,8 \\
\hline Lotes & $\begin{array}{l}\text { SVIS } \\
\text { (índice) }\end{array}$ & $\begin{array}{c}\text { SVIS } \\
\text { (uniform.) }\end{array}$ & $\begin{array}{c}\text { SVIS } \\
\text { (comprim.) }\end{array}$ & $\begin{array}{l}\mathrm{EASS}+\mathrm{SVIS} \\
\quad \text { (indice) }\end{array}$ & $\begin{array}{l}\text { EASS + SVIS } \\
\quad \text { (uniform.) }\end{array}$ & $\begin{array}{l}\text { EASS + SVIS } \\
\text { (comprimento.) }\end{array}$ \\
\hline 1 & $973 \mathrm{~A}$ & $911 \mathrm{~B}$ & $107 \mathrm{AB}$ & $974 \mathrm{~A}$ & $913 \mathrm{~A}$ & $110 \mathrm{~A}$ \\
\hline 2 & $973 \mathrm{~A}$ & $910 \mathrm{~B}$ & $91 \mathrm{~B}$ & 969 A & $896 \mathrm{~A}$ & $91 \mathrm{~B}$ \\
\hline 3 & $975 \mathrm{~A}$ & $918 \mathrm{AB}$ & $113 \mathrm{AB}$ & $975 \mathrm{~A}$ & $917 \mathrm{~A}$ & $109 \mathrm{~A}$ \\
\hline 4 & 979 A & $931 \mathrm{AB}$ & $111 \mathrm{AB}$ & $975 \mathrm{~A}$ & $918 \mathrm{~A}$ & $105 \mathrm{AB}$ \\
\hline 5 & $979 \mathrm{~A}$ & $946 \mathrm{~A}$ & $117 \mathrm{~A}$ & $977 \mathrm{~A}$ & $926 \mathrm{~A}$ & $103 \mathrm{AB}$ \\
\hline C.V.(\%) & 1,0 & 1,9 & 4,2 & 1,9 & 2,3 & 6,2 \\
\hline
\end{tabular}

(*) Comparação de médias dentro de cada coluna (teste de Tukey, $\mathrm{p} \leq 0,05$ ) 
Cabe salientar que as sementes dos dois cultivares apresentavam teor de água inicial entre $8,6 \%$ e 9,0\%. Após o envelhecimento acelerado tradicional, esses valores alcançaram $25,3 \%$ e $27,2 \%$, para as sementes de 'TMG 133RR' e de 22,8\% a 25,3\% para 'Pintado', enquanto após o envelhecimento acelerado em presença de solução saturada de sal, os graus de umidade variaram de $11,6 \%$ a $12,7 \%$; assim, em todos esses casos, não deve ter ocorrido influência do grau de umidade das sementes sobre os resultados dos testes conduzidos (Marcos Filho et al., 2000).

Quanto à identificação apenas dos lotes de menor vigor de ambos os cultivares, deve ser ressaltado que o potencial fisiológico das sementes era muito alto, com germinação elevada ( $\geq 87 \%$ ), de modo que não seria coerente estimar diferenças acentuadas entre os lotes avaliados. Aliás, isto tem acontecido com grande freqüência, em pesquisas recentes sobre testes de vigor de sementes, com várias espécies de grandes culturas e de hortaliças, indicando a eficiência dos procedimentos adotados durante a produção, processamento e armazenamento, como resultado da evolução tecnológica dessa atividade no Brasil.
A interpretação de testes de vigor geralmente depende de decisões tomadas pelo analista, considerando critérios pré-estabelecidos como, por exemplo, os conceitos sobre normalidade de plântulas. No entanto, esse procedimento pode apresentar limitações, destacando-se a variação de resultados entre analistas e laboratórios, face à possível subjetividade da interpretação. Ao mesmo tempo, vários testes consomem período de tempo considerado excessivo para a obtenção dos resultados. Conseqüentemente, a automatização desses procedimentos, via recursos computacionais, pode contribuir significativamente para aprimorar a precisão de resultados, além da obtenção mais rápida da informação.

Os resultados apresentados nas Tabelas 1 e 2 indicam que a análise computadorizada $\mathrm{SVIS}^{\circledR}$ não detectou diferenças significativas entre os índices de vigor calculados para os lotes de 'TMG 113RR', tanto após a germinação como para o envelhecimento acelerado com solução de sal, nas duas épocas de testes; a uniformidade de desenvolvimento também não mostrou diferenças consistentes entre os lotes. Essas mesmas observações se aplicam para as sementes do cultivar Pintado (Tabelas 3 a 5).

TABELA 3. Valores médios obtidos em testes de germinação, primeira contagem de germinação, envelhecimento acelerado tradicional e com solução saturada de sal, emergência de plântulas e índices de vigor, uniformidade e comprimento de plântulas de soja determinados em análises SVIS (após germinação e envelhecimento acelerado) de cinco lotes de sementes de soja, cultivar Pintado, na primeira época experimental. Piracicaba, SP, 2008.

\begin{tabular}{|c|c|c|c|c|c|c|}
\hline Lotes & Germinação (\%) & \multicolumn{2}{|c|}{$1^{\mathrm{a}}$ Contagem $(\%)$} & EAT 48 h (\%) & EASS 72 h (\%) & Emergência de Plântulas (\%) \\
\hline 6 & $93 \mathrm{~A}^{(*)}$ & \multicolumn{2}{|c|}{$91 \mathrm{~A}$} & $88 \mathrm{~A}$ & $94 \mathrm{~A}$ & $92 \mathrm{~A}$ \\
\hline 7 & $95 \mathrm{~A}$ & \multicolumn{2}{|c|}{$93 \mathrm{~A}$} & $90 \mathrm{~A}$ & $95 \mathrm{~A}$ & $96 \mathrm{~A}$ \\
\hline 8 & $97 \mathrm{~A}$ & \multicolumn{2}{|c|}{$94 \mathrm{~A}$} & $86 \mathrm{~A}$ & $94 \mathrm{~A}$ & $95 \mathrm{~A}$ \\
\hline 9 & $94 \mathrm{~A}$ & \multicolumn{2}{|c|}{$92 \mathrm{~A}$} & $87 \mathrm{~A}$ & $93 \mathrm{~A}$ & $97 \mathrm{~A}$ \\
\hline 10 & $89 \mathrm{~A}$ & \multicolumn{2}{|c|}{$86 \mathrm{~A}$} & $82 \mathrm{~A}$ & $89 \mathrm{~A}$ & $94 \mathrm{~A}$ \\
\hline C.V. (\%) & 5,5 & \multicolumn{2}{|c|}{5,9} & 7,4 & 6,4 & 5,5 \\
\hline Lotes & $\begin{array}{l}\text { SVIS } \\
\text { (índice) }\end{array}$ & $\begin{array}{c}\text { SVIS } \\
\text { (uniform.) }\end{array}$ & $\begin{array}{c}\text { SVIS } \\
\text { (comprim.) }\end{array}$ & $\begin{array}{c}\text { EASS + } \\
\text { SVIS } \\
\text { (indice) }\end{array}$ & $\begin{array}{c}\text { EASS + } \\
\text { SVIS } \\
\text { (uniform.) }\end{array}$ & $\begin{array}{l}\text { EASS + SVIS } \\
\text { (comprimento.) }\end{array}$ \\
\hline 6 & $975 \mathrm{~A}$ & $918 \mathrm{~A}$ & $76 \mathrm{~A}$ & $973 \mathrm{~A}$ & $911 \mathrm{~A}$ & $74 \mathrm{~A}$ \\
\hline 7 & $961 \mathrm{~A}$ & $912 \mathrm{~A}$ & $69 \mathrm{AB}$ & $975 \mathrm{~A}$ & $918 \mathrm{~A}$ & $72 \mathrm{~A}$ \\
\hline 8 & $975 \mathrm{~A}$ & $919 \mathrm{~A}$ & $72 \mathrm{AB}$ & $970 \mathrm{~A}$ & $901 \mathrm{~A}$ & $70 \mathrm{~A}$ \\
\hline 9 & $968 \mathrm{~A}$ & $898 \mathrm{~A}$ & $64 \mathrm{BC}$ & $973 \mathrm{~A}$ & $912 \mathrm{~A}$ & $74 \mathrm{~A}$ \\
\hline 10 & $936 \mathrm{~A}$ & $912 \mathrm{~A}$ & $60 \mathrm{C}$ & $975 \mathrm{~A}$ & $918 \mathrm{~A}$ & $67 \mathrm{~B}$ \\
\hline C.V.(\%) & 2,4 & 1,4 & 5,9 & 1,3 & 1,1 & 5,2 \\
\hline
\end{tabular}

(*) Comparação de médias dentro de cada coluna (teste de Tukey, $\mathrm{p} \leq 0,05$ ) 
TABELA 4. Valores médios obtidos referentes a índices de vigor, uniformidade e comprimento de plântulas de soja determinados em análises SVIS (após germinação e envelhecimento acelerado) de cinco lotes de sementes de soja, cultivar Pintado, na segunda época experimental. Piracicaba, SP, 2008.

\begin{tabular}{ccccccc}
\hline Lotes & $\begin{array}{c}\text { SVIS } \\
\text { (índice) }\end{array}$ & $\begin{array}{c}\text { SVIS } \\
\text { (uniform.) }\end{array}$ & $\begin{array}{c}\text { SVIS } \\
\text { (comprim.) }\end{array}$ & $\begin{array}{c}\text { EASS + SVIS } \\
\text { (indice) }\end{array}$ & $\begin{array}{c}\text { EASS + SVIS } \\
\text { (uniform.) }\end{array}$ & $\begin{array}{c}\text { EASS + SVIS } \\
\text { (comprimento. })\end{array}$ \\
\hline 6 & $973 \mathrm{~A}^{(*)}$ & $913 \mathrm{~A}$ & $94 \mathrm{~A}$ & $976 \mathrm{~A}$ & $920 \mathrm{~A}$ & $94 \mathrm{~A}$ \\
7 & $977 \mathrm{~A}$ & $924 \mathrm{~A}$ & $88 \mathrm{~A}$ & $975 \mathrm{~A}$ & $920 \mathrm{~A}$ & $95 \mathrm{~A}$ \\
8 & $976 \mathrm{~A}$ & $920 \mathrm{~A}$ & $85 \mathrm{~A}$ & $977 \mathrm{~A}$ & $923 \mathrm{~A}$ & $91 \mathrm{~A}$ \\
9 & $975 \mathrm{~A}$ & $920 \mathrm{~A}$ & $93 \mathrm{~A}$ & $977 \mathrm{~A}$ & $925 \mathrm{~A}$ & $79 \mathrm{~A}$ \\
10 & $977 \mathrm{~A}$ & $925 \mathrm{~A}$ & $80 \mathrm{~B}$ & $974 \mathrm{~A}$ & $916 \mathrm{~A}$ & 5,3 \\
\hline C.V.(\%) & 1,4 & 1,3 & 7,4 & 1,7 & 1,3 & \\
\hline
\end{tabular}

(*) Comparação de médias dentro de cada coluna (teste de Tukey, $\mathrm{p}<0,05$ )

TABELA 5. Valores médios obtidos em testes de germinação, envelhecimento acelerado com solução saturada de sal, emergência de plântulas e índices de vigor, uniformidade e comprimento de plântulas de soja determinados em análises SVIS (após germinação e envelhecimento acelerado) de cinco lotes de sementes de soja, cultivar Pintado, na terceira época experimental. Piracicaba, SP, 2008.

\begin{tabular}{|c|c|c|c|c|c|c|}
\hline Lotes & Germinação (\%) & \multicolumn{2}{|c|}{$1^{\underline{a}}$ Contagem $(\%)$} & EASS $72 \mathrm{~h}(\%)$ & \multicolumn{2}{|c|}{ Emergência de Plântulas (\%) } \\
\hline 6 & $93 \mathrm{AB}^{(*)}$ & \multicolumn{2}{|c|}{$90 \mathrm{~A}$} & \multicolumn{2}{|l|}{$93 \mathrm{~A}$} & $96 \mathrm{~A}$ \\
\hline 7 & $93 \mathrm{AB}$ & \multicolumn{2}{|c|}{$85 \mathrm{~A}$} & \multicolumn{2}{|l|}{$93 \mathrm{~A}$} & $91 \mathrm{~A}$ \\
\hline 8 & $96 \mathrm{~A}$ & \multicolumn{2}{|c|}{$94 \mathrm{~A}$} & \multicolumn{2}{|c|}{$88 \mathrm{AB}$} & $93 \mathrm{~A}$ \\
\hline 9 & $92 \mathrm{AB}$ & \multicolumn{2}{|c|}{$89 \mathrm{~A}$} & \multicolumn{2}{|c|}{$92 \mathrm{AB}$} & $97 \mathrm{~A}$ \\
\hline 10 & $87 \mathrm{~B}$ & \multicolumn{2}{|c|}{$79 \mathrm{~B}$} & \multicolumn{2}{|l|}{$84 \mathrm{~B}$} & $82 \mathrm{~B}$ \\
\hline C.V. (\%) & 6,7 & \multicolumn{2}{|r|}{4,2} & 5,5 & \multicolumn{2}{|c|}{8,1} \\
\hline Lotes & $\begin{array}{c}\text { SVIS } \\
\text { (índice) }\end{array}$ & $\begin{array}{c}\text { SVIS } \\
\text { (uniform.) }\end{array}$ & $\begin{array}{c}\text { SVIS } \\
\text { (comprim.) }\end{array}$ & $\begin{array}{l}\text { EASS + SVIS } \\
\text { (índice) }\end{array}$ & $\begin{array}{l}\text { EASS + SVIS } \\
\text { (uniform.) }\end{array}$ & $\begin{array}{l}\text { EASS + SVIS } \\
\text { (comprimento.) }\end{array}$ \\
\hline 6 & $978 \mathrm{~A}$ & $927 \mathrm{~A}$ & $96 \mathrm{~A}$ & $974 \mathrm{~A}$ & $916 \mathrm{~A}$ & $95 \mathrm{~A}$ \\
\hline 7 & $976 \mathrm{~A}$ & $921 \mathrm{~A}$ & $91 \mathrm{~A}$ & $976 \mathrm{~A}$ & $921 \mathrm{~A}$ & $86 \mathrm{AB}$ \\
\hline 8 & $979 \mathrm{~A}$ & $932 \mathrm{~A}$ & $93 \mathrm{~A}$ & $976 \mathrm{~A}$ & $917 \mathrm{~A}$ & $84 \mathrm{AB}$ \\
\hline 9 & $980 \mathrm{~A}$ & $934 \mathrm{~A}$ & $97 \mathrm{~A}$ & $978 \mathrm{~A}$ & $929 \mathrm{~A}$ & $88 \mathrm{AB}$ \\
\hline 10 & $976 \mathrm{~A}$ & $922 \mathrm{~A}$ & $82 \mathrm{~B}$ & $973 \mathrm{~A}$ & $910 \mathrm{~A}$ & $72 \mathrm{~B}$ \\
\hline C.V.(\%) & 1,2 & 1,7 & 3,9 & 1,7 & 1,4 & 6,2 \\
\hline
\end{tabular}

(*) Comparação de médias dentro de cada coluna (teste de Tukey, $\mathrm{p} \leq 0,05$ )

A Ohio Crop Improvement Association, entidade certificadora de sementes no estado de Ohio/EUA, realizando análises SVIS $^{\circledR}$ em amostras sementes de soja, desenvolveu uma escala em que sementes de alto vigor originam índices de 800 a 1000 após germinação durante 3 dias, enquanto valores de 500 a 1000 são registrados para essas sementes após o envelhecimento acelerado. As de baixo vigor geram valores inferiores a $300 \mathrm{em}$ ambas as situações (Comunicação Pessoal). Conseqüentemente, se os valores médios correspondentes aos índices de vigor, com alta freqüência, foram superiores a 950, pode-se considerar que os lotes aqui examinados seriam classificados como de "alto vigor", de modo que apenas parâmetros mais sensíveis poderiam ser capazes de detectar diferenças significativas entre os lotes.

Sekharan (2005), trabalhando com lotes de sementes de soja envelhecidas artificialmente, variando entre $83 \%$ e 95\% de germinação, constatou a eficiência de análises $\mathrm{SVIS}^{\circledR}$ para detectar diferenças no potencial fisiológico. Assim, os parâmetros índice de vigor e uniformidade de desenvolvimento podem ser úteis quando as variações entre lotes forem mais acentuadas que as verificadas na presente 
pesquisa. Informações semelhantes foram relatadas por Otoni et al. (2008), com sementes de algodão.

Embora haja vários métodos sejam disponíveis para determinar o vigor de sementes, um dos mais importantes é a avaliação da velocidade e uniformidade de crescimento, enfatizada na própria conceituação de vigor. A sensibilidade desse parâmetro para identificar diferenças no potencial fisiológico de sementes dos dois cultivares, após as análises SVIS $^{\circledR}$, ficou evidenciada pelo exame das Tabelas 1 a 5. Para os dois cultivares, os dados médios do comprimento de plântulas com 3 dias de idade, provenientes do teste de germinação ou da germinação após o envelhecimento acelerado, indicaram a inferioridade do vigor do lote 2 de 'TMG 113RR' e do lote 10 de 'Pintado'. Além de consistentes, esses resultados são comparáveis aos obtidos nos testes de envelhecimento tradicional e de envelhecimento acelerado com solução saturada de sal, considerados eficientes para a determinação do vigor de sementes de soja (Marcos Filho et al., 2000).

O teste de comprimento de plântulas ou de suas partes tem sido consideradoeficiente para detectardiferenças no potencial fisiológico de sementes de várias espécies (Nakagawa, 1999). Ao mesmo tempo, além dessa sensibilidade, seus resultados podem apresentar estreita relação com a emergência de plântulas em campo (Krzyzanowski, 1991; Vanzolini et al., 2007). Essas observações foram confirmadas nas análises automatizadas SVIS $^{\circledR}$, pois o comprimento de plântulas foi um parâmetro mais sensível para indicar diferenças de vigor entre os lotes que o índice de vigor e a uniformidade de desenvolvimento.

Embora a presente pesquisa não tenha incluído comparação com o procedimento manual para determinação do comprimento de plântulas, há possibilidade de uma subestimativa pela análise SVIS ${ }^{\circledR}$ porque o software analisa apenas uma imagem bidimensional de cada plântula. Assim, enquanto esta geralmente é "esticada" antes da mensuração com auxílio de régua, o software demarca cada plântula, respeitando possíveis curvaturas, como mostram as figuras apresentadas no texto; essas curvaturas não permitem a avaliação exata do comprimento da plântula, mas esses desvios são pouco expressivos e não diminuem a vantagem considerável da utilização de processo automatizado.

Carneiro e Guedes (2002) destacaram que a interpretação do teste de envelhecimento acelerado para avaliação do potencial fisiológico tem sido dirigida principalmente ao cômputo da percentagem final de germinação (plântulas normais), cujas médias podem ser relacionadas ou não com os resultados de outros testes conduzidos após um determinado período de armazenamento das sementes ou com a emergência de plântulas em campo. Segundo esses autores, os resultados do envelhecimento acelerado geralmente são traduzidos pelo grau de tolerância às condições adversas de temperatura e umidade relativa, expressos principalmente pela sobrevivência das sementes e não necessariamente pela queda das taxas de reações químicas que determinam a velocidade de germinação e a taxa de crescimento de plântulas, ou seja, eventos da deterioração que precedem a morte das sementes. Conseqüentemente, sugeriram estudos dirigidos à determinação da possibilidade da interpretação do teste de envelhecimento acelerado mediante a avaliação da velocidade de germinação e do crescimento médio de plântulas, complementando o procedimento tradicional de determinação da percentagem de plântulas normais.

Com base nessas considerações, a utilização do SVIS ${ }^{\circledR}$ para avaliação da resposta de diferentes lotes de sementes ao envelhecimento acelerado, pode ser considerada como alternativa promissora e que merece consideração em pesquisas futuras. Esse sistema representa um procedimento simples, reproduzível e objetivo para avaliação direta do vigor de sementes germinadas ou como ferramenta suplementar para a interpretação de outros testes, como envelhecimento acelerado, germinação a baixa temperatura, crescimento de plântulas, velocidade de germinação ou mesmo para identificar efeitos de tratamentos químicos, do condicionamento fisiológico e outras situações em que é interessante o monitoramento da germinação e do crescimento de plântulas em intervalos regulares.

O período necessário para a análise SVIS $^{\circledR}$ de cada amostra, cerca de 4 a 5 minutos, poderia ser reduzido significativamente, não fosse a necessidade da transferência das plântulas do papel-toalha (coloração branca) para a folha de cartolina azul escuro, para a obtenção de contraste adequado para a análise de imagens das plântulas. Assim, a disponibilidade de papel-toalha de coloração marrom, como nos EUA, certamente permitiria a análise de 40 amostras de 25 plântulas, desde a captação de imagens pelo scanner, em aproximadamente 100 minutos (Marcos Filho et al., 2006).

Desta maneira, os resultados desta pesquisa proporcionaram resultados interessantes a respeito da determinação do potencial fisiológico de sementes de soja. As análises automatizadas SVIS $^{\circledR}$ permitiram identificar diferenças de vigor entre lotes de sementes com poder germinativo elevado, de maneira comparável aos resultados de outros testes recomendados pela pesquisa. Assim, o sistema também pode ser considerado promissor para outras espécies. O software desenvolvido para soja pode ser utilizado para feijão e outras leguminosas em que a estrutura 
da plântula é semelhante. Há opções disponíveis para alface (Sako et al., 2001), cucurbitáceas (Marcos Filho et al., 2006), milho (Hoffmaster et al., 2005; Otoni e McDonald, 2005) e algodão (Otoni et al., 2008), mas a pesquisa sobre o assunto tem sido dinâmica e novos usos certamente serão divulgados a curto prazo.

\section{CONCLUSÕES}

A análise dos dados e a interpretação dos resultados do presente trabalho permitiram concluir que a análise computadorizada de imagens de plântulas provenientes do teste de germinação ou do envelhecimento acelerado com solução saturada de sal, com o uso do software SVIS ${ }^{\circledR}$, é sensível para a determinação do vigor de sementes de soja. Essa sensibilidade é mais acentuada quando o sistema é utilizado para a avaliação do crescimento de plântulas.

\section{REFERÊNCIAS}

BRASIL, Ministério da Agricultura e Reforma Agrária. Regras para análise de sementes. Brasília, DF: SNDA/ DNDV/CLAV, 1992. $365 \mathrm{p}$.

CARNEIRO, J.W.P.; GUEDES, T.A. Dinâmica de ocorrências germinativas em amostras de sementes envelhecidas artificialmente. Informativo ABRATES, v. 12, n. 1,2,3, p. 44-51, 2002.

COSTA, N.P.; MARCOS FILHO, J.; FRANÇA NETO, J. DE B.; KRZYZANOWSKI, F.C.; HENNING, A.A. Teste de tetrazólio em semente de soja com preocondicionamento abreviado - Série Sementes. Londrina: Embrapa Soja, 2008, 7p. (Embrapa Soja. Circular Técnica, 56).

HOFFMASTER , A.L.; FUJIMURA, K.; MCDONALD, M.B.; BENNETT, M.A. Na automated system for vigor testing three-day old soybean seedlings. Seed Science and Technology, v. 31, n.3, p. 701-713, 2003.

HOFFMASTER, A. F.; XU, L.; FUJIMURA, K.; MCDONALD, M.B.; BENNETT, M.A.; EVANS, A.F. The Ohio State University seed vigor imaging system (SVIS) for soybean and corn seedlings. Seed Technology, v. 27, n.1, p. 7-24, 2005.

KRZYZANOWSKI, F.C. Teste de comprimento de raiz de plântula de soja. Informativo ABRATES, v. 2, n. 1, p. 1114, 1991.

MARCOS FILHO, J. Conceitos e testes de vigor para sementes de soja. CONGRESSO BRASILEIRO DE SOJA, 1., 1999, Londrina. Londrina: EMBRAPA-CNPSo, 1999.
Anais... p.220-226, 1999a.

MARCOS FILHO, J. Testes de vigor: importância e utilização. In: KRZYZANOWSKI, F.C.; VIEIRA, R.D.; FRANÇA NETO, J.B. (Ed.). Vigor de sementes: conceitos e testes. Londrina: ABRATES, 1999b. p.1.1-1.24

MARCOS FILHO, J.; NOVEMBRE, A.D.L.C.; CHAMMA, H.M.C.P. Tamanho da semente e teste de envelhecimento acelerado para soja. Scientia Agricola, v. 57, n.3, p. 473482, 2000.

MARCOS FILHO, J.; BENNETT, M.A.; MCDONALD, M.B.; EVANS, A.F.; GRASSBAUGH, E.M. Assessment of melon seed vigour by na automated computer imaging system compared to traditional procedures. Seed Science and Technology, v. 34, n.2, p. 485-497, 2006.

MCCORMAC, A.C.; KEEFE, P.D.; DRAPER, S.R. Cauliflower seed vigor: imbibitions effects. Journal of Experimental Botany, v. 41, n. 4, p. 893-899, 1990.

MCDONALD, M.B.; FUJIKURA, K; SAKO, Y; EVANS, A.F.; BENNETT, M.A. Computer imaging to improve seed quality determinations. In: Digital imaging and spectral techniques: application to precision agriculture and crop physiology. ASA Special Publication, n.66, p.15-27, 2003.

NAKAGAWA, J. Testes de vigor baseados no desempenho das plântulas. In: KRZYZANOWSKI, F.C.; VIEIRA, R.D.; FRANÇA NETO, J.B. (Ed.). Vigor de sementes: conceitos e testes. Londrina: ABRATES, 1999. p.2.1-2.24.

OTONI, R.R.; MCDONALD, M.B. Moisture and temperature effects on maize and soybean seedlings using the seed vigor imaging system. Seed Technology, v. 27, n. 2, p.243-247, 2005.

OTONI, R.R.; MCDONALD, M.B.; TAY, D. The use of $\mathrm{X}$-Rays for seed vigor classification of cotton seeds. Seed Technology, 2008 (submetido).

SAKO, Y.; MCDONALD, M.B.; FUJIMURA, K.; EVANS, A.F.; BENNETT, M.A. A system for automated seed vigour assessment. Seed Science and Technology, v. 29, n. 3, p. 625-636, 2001.

SEKHARAN, S. Aged soybean seeds - their physiology and vigor assessment. 2006. $180 \mathrm{f}$. Tese (Ph.D) - The Ohio State University, Columbus/OH/EUA, 2005.

STEINER, J.J. Seedling rate of development index: indicator of vigor and seedling growth response. Crop Science, v. 30, n.6, p. 1264-1271, 1990.

VANZOLINI, S.;ARAKI, C.A.S.; SILVA, A.C.M.T.; NAKAGAWA, J. Teste de comprimento de plântula na 
avaliação da qualidade fisiológica de sementes de soja. Revista Brasileira de Sementes, v. 29, n. 2, p. 90-96, 2007.

VIEIRA, R.D..Testes de vigor utilizados para sementes de soja no Brasil na atualidade. CONGRESSO BRASILEIRO DE SOJA, 1., Londrina, 1999. Londrina: EMBRAPACNPSo, 1999. Anais... 1999. p.227-232
VIEIRA, R.D.; BITTENCOURT, S.R.M.; PANOBIANCO, $M$. Seed vigour - an important component of seed quality in Brazil. ISTA - Seed Testing International, n. 126, p. 21-22, 2003.

WOODSTOCK, L.W. Seedling growth as a measure of seed vigor. Proceedings of International Seed Testing Association, v. 34, n. 2, p. 273-280, 1969. 